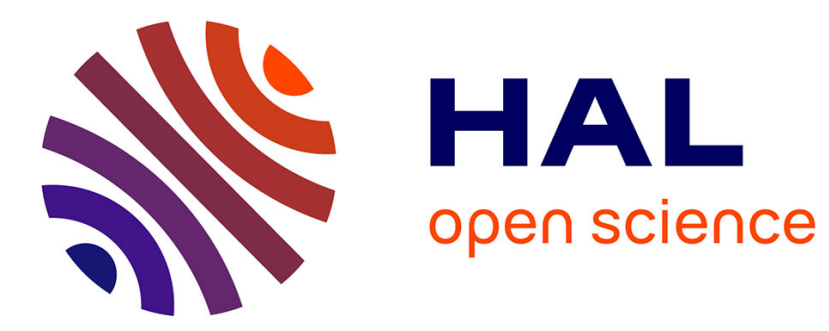

\title{
Les archives Roger Schneider (1917-2002) au centre Walda Masqal (Institute of Ethiopian Studies, Addis Abeba)
}

\author{
Marie-Laure Derat
}

\section{- To cite this version:}

Marie-Laure Derat. Les archives Roger Schneider (1917-2002) au centre Walda Masqal (Institute of Ethiopian Studies, Addis Abeba). Annales d'Éthiopie, 2011, 26 (1), pp.291-302. 10.3406/ethio.2011.1443 . halshs-02552676

\section{HAL Id: halshs-02552676 \\ https://shs.hal.science/halshs-02552676}

Submitted on 23 Apr 2020

HAL is a multi-disciplinary open access archive for the deposit and dissemination of scientific research documents, whether they are published or not. The documents may come from teaching and research institutions in France or abroad, or from public or private research centers.
L'archive ouverte pluridisciplinaire HAL, est destinée au dépôt et à la diffusion de documents scientifiques de niveau recherche, publiés ou non, émanant des établissements d'enseignement et de recherche français ou étrangers, des laboratoires publics ou privés. 


\section{Les archives Roger Schneider (1917-2002) au centre Walda Masqal (Institute of Ethiopian Studies, Addis Abeba)}

\section{Marie-Laure Derat}

\section{Abstract}

The archives of Roger Schneider (1917-2002) in the Walda Masqal Centre (Institute of Ethiopian Studies, Addis Ababa) The archives of Roger Schneider are now available in the Walda Masqal centre, an archives' centre of the Institute of Ethiopian Studies in Addis Abeba. This article presents the documents of this collection, i. e. the library itself given by the family of Roger Schneider, as well as the archives of this great scholar, which have been digitalised in order to preserve them. This digitalisation was the result of a joint project between the Centre Français des Études Éthiopiennes and the Institute of Ethiopian Studies.

\section{Résumé}

Les archives Roger Schneider sont aujourd'hui consultables au centre Walda Masqal, centre d'archives de l'Institute of Ethiopian Studies à Addis Abeba. Cet article présente le fonds documentaire, aussi bien la bibliothèque donnée par la famille de Roger Schneider, que les archives du savant, qui ont été numérisées dans le cadre d'un projet conjoint au Centre Français des Études Éthiopiennes et à l'Institute of Ethiopian Studies, afin de les sauvegarder.

\section{Citer ce document / Cite this document :}

Derat Marie-Laure. Les archives Roger Schneider (1917-2002) au centre Walda Masqal (Institute of Ethiopian Studies, Addis Abeba). In: Annales d'Ethiopie. Volume 26, année 2011. pp. 291-302;

doi : $10.3406 /$ ethio.2011.1443

http://www.persee.fr/doc/ethio_0066-2127_2011_num_26_1_1443

Document généré le 08/03/2018 


\title{
Les archives Roger Schneider (1917-2002) au centre Walda Masqal (Institute of Ethiopian Studies, Addis Abeba)
}

\author{
Marie-Laure Derat ${ }^{*}$
}

Après des études de linguistique, notamment auprès de Marcel Cohen, Roger Schneider entre au CNRS en 1951. Il rejoint la Mission archéologique française à Addis Abeba à partir de 1957, en qualité d'épigraphiste et de philologue. La section d'archéologie, installée à proximité des archives nationales d'Éthiopie, accueillait alors des experts français chargés de "promouvoir et développer les études et recherches archéologiques en Éthiopie $»^{1}$. Cette section fut l'ancêtre à la fois du Centre Français des Études Éthiopiennes (CFEE) et de l'Authority for Research and Conservation of Cultural Heritage ${ }^{2}$, service du ministère éthiopien de la culture, en charge du patrimoine. À sa retraite en 1982, Roger Schneider enseigne l'histoire et l'épigraphie de l'Éthiopie antique à l'Université d'Addis Abeba et ce pendant une quinzaine d'années ${ }^{3}$.

Roger Schneider était un savant, comme on pouvait encore en rencontrer au $\mathrm{XX}^{\mathrm{e}}$ siècle. Épigraphiste, linguiste, philologue, il connaissait l'amharique, le ge'ez l'arabe, le sabéen et l'assyrien en plus de nombreuses langues européennes. Il s'est notamment intéressé à l'origine et à l'évolution de l'écriture éthiopienne. Parmi ses nombreuses publications, l'une des plus importantes est sans aucun doute celle qu'il a préparée avec Abraham Johannes Drewes - le Recueil des inscriptions de l'Éthiopie des périodes préaxoumites et axoumites ${ }^{4}$ - catalogue présentant les documents, les planches et les transcriptions des toutes les inscriptions sabéennes, proto-ge'ez, ge'ez et grecques (étudiées par Étienne Bernand), en Éthiopie. Malheureusement, le dernier

\footnotetext{
Centre Français des Études Éthiopiennes (USR 3137/UMIFRE 23), CNRS \& Ministère des Affaires Étrangères, Addis Abeba, Éthiopie.

1 Kebbede Mikael \& Leclant, $1955: 1$.

2 Le contexte de la création de la section d'archéologie au sein de l'Institut éthiopien d'Études et de Recherches a été bien retracé par Chekroun, 2011. Pour une présentation de la mission archéologique française, voir aussi Kebbede Mikael \& Leclant, 1955.

3 La carrière scientifique de Roger Schneider a été retracée, à sa mort, par ses collègues et amis. Voir Drewes, 2002 ; Anfray, 2002, 2003.

4 Bernand, Drewes, Schneider, 1991 ; Bernand, 2000.
} 


\section{Marie-Laure Derat}

volume traitant de la traduction des inscriptions en langue ge'ez n'était pas achevé à sa mort et n'a toujours pas été publié5.

À la mort de Roger Schneider en avril 2002, sa famille a choisi de faire don de sa bibliothèque et de ses archives personnelles à l'Institute of Ethiopian Studies (IES) à Addis Abeba. Ce dernier a alors stocké la quarantaine de cartons abritant tout le matériel retraçant la carrière intellectuelle de ce savant dans un centre d'archives, l'ancienne maison d'un dignitaire du régime impérial, Walda Masqal, sise dans le quartier de Kazanchis. Ce centre conserve en particulier les archives de l'ancien Ministère de la réforme foncière ainsi que celles du Département du régime foncier de l'ancien ministère éthiopien de l'intérieur.

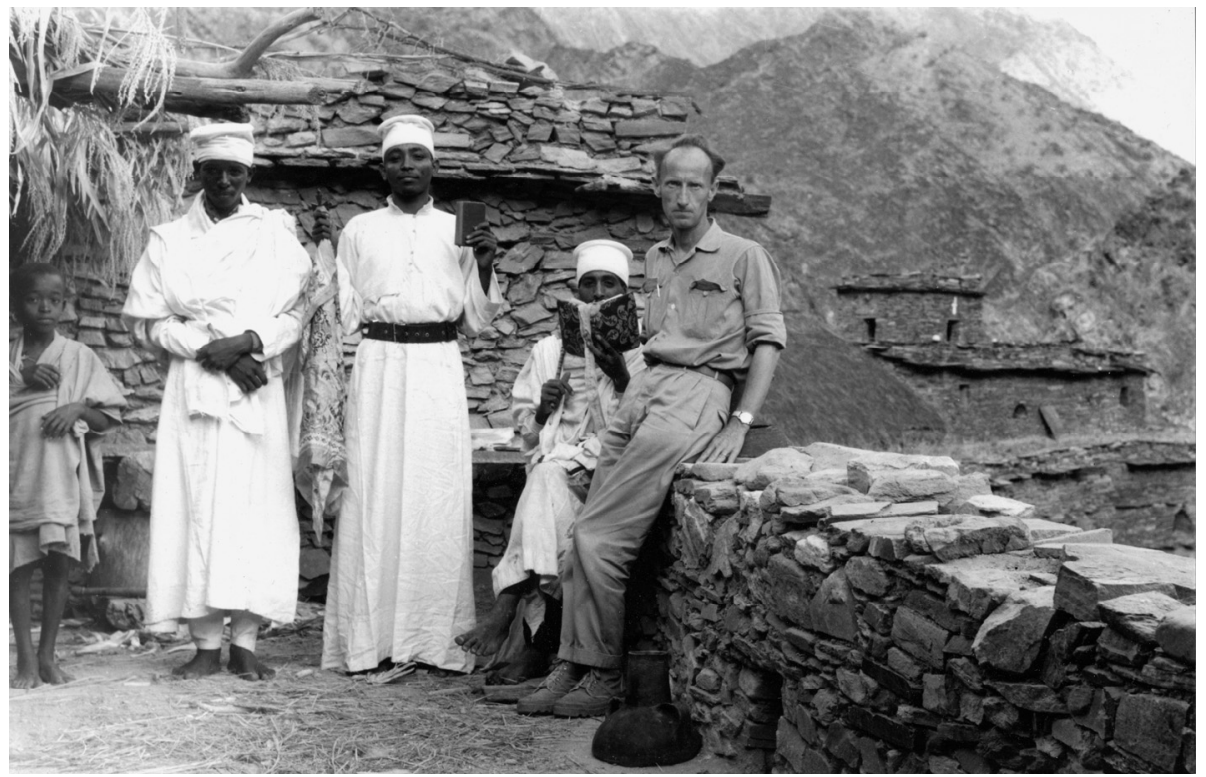

Roger Schneider lors de l'une de ses visites au monastère de Gunda Gundē (années 1960)

Jusqu'en 2010, les archives de Roger Schneider sont restées en carton et commençaient à être menacées, la pièce où elles étaient stockées n'étant pas étanche lors des pluies saisonnières. Une partie des cartons prenait l'humidité. Intéressée par les travaux de Roger Schneider sur l'évangéliaire de Dabra Libānos de Hām et souhaitant consulter ses archives sur le sujet, j’ai proposé à la bibliothèque de l'IES et à son directeur, Ato Daniel Mamo, de classer, inventorier (en anglais) et numériser ces archives afin de les sauvegarder et de les mettre à la disposition de

5 C'est Abraham Drewes qui a alors poursuivi le travail (Drewes, $2002: 210$; Leclant, 2002 : 290), mais n'a pas pu présider à sa publication avant sa mort en 2007. Le manuscrit du dernier volume du Recueil des inscriptions de l'Étbiopie des périodes préaxoumites et axoumites est aujourd'hui entre les mains de Christian Robin et d'Iwona Gajda qui, on l'espère, seront en mesure de mener à bien la finalisation de cet important projet. 
tout à chacun. Ce projet, débuté à l'été 2010, s'est achevé à l'automne 20116. Le propos de cet article est donc de donner quelques éléments d'information concernant les archives Roger Schneider, afin de livrer un aperçu de ce qu'elles contiennent et de ce qu'elles ne contiennent pas et de faciliter leur consultation, voire leur traitement.

Documents papiers, photographies, films négatifs ont été numérisés sur place à l'aide de scanners. Les quelques bandes audio retrouvées ont été numérisées par un laboratoire en France, spécialisé dans la restauration audio. Dans la mesure du possible, l'ordre des documents a été respecté voir reconstitué - de nombreux dossiers ayant été dispersés. S’agissant des développements de photographies sur papier, quand une mention permettant l'identification était inscrite au dos, le dos a également été scanné. Il n'en reste pas moins que de nombreux documents n'ont pas été identifiés faute d'informations et du fait du désordre intervenu dans certains dossiers, probablement au cours du déménagement.

Il reste encore de nombreuses choses à faire concernant les archives. Le projet était avant tout de sauvegarder la documentation avant qu'elle soit totalement endommagée par l'humidité. Le classement opéré respecte autant que faire se peut le classement réalisé par Roger Schneider lui-même. Mais pour que ces archives soient exploitées par les chercheurs, il faudrait notamment identifier les nombreuses inscriptions photographiées et étudiées par Roger Schneider, tout comme certains manuscrits, ou sites, ce qui n'est pas toujours le cas en l'état actuel. Il faudrait aussi compter le nombre de pièces constitutives de chaque dossier, en fournir une description rapide. Bref, réaliser un véritable travail d'archiviste.

\section{La bibliothèque de Roger Schneider}

L'essentiel du fonds est composé de la bibliothèque personnelle de Roger Schneider, comprenant des livres, revues, tirés à part, copies de publications scientifiques sur différents supports. Ce sont, en tout, un peu plus de 200 ouvrages, 25 revues et près de 500 articles qui constituent cette bibliothèque. Ouvrages et revues sont désormais classés par ordre alphabétique et rangés sur des étagères dans l'un des bureaux du centre Walda Masqal, tandis que les articles ont été placés dans des boîtes d'archives, également par ordre alphabétique des auteurs. Un inventaire de l'ensemble, papier et numérique, est accessible au centre Walda Masqal et permet de trouver le plus rapidement possible l'information nécessaire aux chercheurs.

Le contenu de cette bibliothèque témoigne des centres d'intérêt de Roger Schneider et de ses liens privilégiés avec d'autres chercheurs. On trouve ainsi de nombreux travaux de Marcel Cohen et David Cohen, Abraham Johannes Drewes, Rodolfo Fattovich, Walter Müller, Karen Petráček, Christian Robin, Gonzague Ryckmans et Jacques Ryckmans ou encore Stefan Strelcyn. Cette liste n'est bien sûr

\footnotetext{
6 Ce travail est le fruit d'une collaboration entre le CFEE et l'IES. Je tiens ici à remercier tous ceux qui ont participé au classement et à la numérisation des archives : au centre Walda Masqal, Tabi Asfaw, Mimi Yohannes et Mesfin Legesse ; au CFEE, Tensae Eskender et Mehdi Lebzaë.
} 


\section{Marie-Laure Derat}

pas exhaustive, mais elle souligne la position de Roger Schneider au sein de la communauté des sémitisants.

Parmi les ouvrages inventoriés, il faut aussi signaler un très grand nombre de livres publiés par la mission catholique d'Asmara ou la mission évangélique d'Asmara. Ainsi, les travaux concernant la langue Kunama, destinés à l'évangélisation des Kunama, rassemblent une grammaire, un vocabulaire, des textes bibliques et un catéchisme en Kunama. En revanche, l'ouvrage de référence de Leo Reinisch ne figure pas dans la collection.

Le fonds de l'IES s'est ainsi enrichi d'une bibliothèque spécialisée, d'un grand intérêt pour les étudiants et chercheurs éthiopiens qui n'ont pas toujours facilement accès à la documentation, qui plus est quand celle-ci est éparpillée dans de nombreuses revues peu diffusées.

\section{Les archives}

Quant aux archives proprement dites, elles consistent essentiellement en dossiers thématiques organisés par Roger Schneider en fonction des sujets sur lesquels il travaillait. Par exemple, on trouve des dossiers intitulés: traité sur l'antéchrist d'Hippolyte de Rome ${ }^{7}$, Enda Qirqos, épigraphie aksumite préchrétienne, écriture, MKRB, etc. Dans ces dossiers, figurent des photocopies d'articles, des notes personnelles de Roger Schneider sur la littérature consultée, des photographies d'inscriptions, de manuscrits, de monuments, des traductions et des brouillons d'articles de Roger Schneider. Ce sont ces dossiers qui ont été numérisés car il s'agit le plus souvent de notes prises sur des papiers fragiles, parfois écrites au crayon papier, accompagnées de photographies développées sur papier dont la qualité s'estompe dans le temps. Pour la numérisation, il a fallu faire des choix : il n'y avait aucun intérêt à numériser les tirés à part ou photocopies d'articles publiés. Et dans la mesure où ces publications étaient parties prenantes de la bibliothèque de Roger Schneider, elles ont été sorties des dossiers thématiques pour être intégrées au reste de la documentation publiée et classées par ordre alphabétique. Si bien que n'ont été numérisés que les documents qui n'étaient pas des publications, à l'exception des premières versions d'articles publiés ensuite, brouillons corrigés de la main de Roger Schneider.

Parmi les dossiers d'archives, figuraient également des dossiers nominatifs, dédiés aux savants avec lesquels Roger Schneider était régulièrement en contact et avec lesquels il échangeait une correspondance, qui accompagnait souvent l'envoi de tirés à part. D’où, dans les dossiers classés et numérisés, des intitulés comme W. Hahn ou W.W. Müller. Pour le cas où la correspondance était limitée ou associée à un thème de recherche particulier, bien souvent elle est intégrée dans des

\footnotetext{
7 Ce dossier n'apporte pas grand-chose sur le traité lui-même, manuscrit découvert à Gunda Gundē en 1962 par Roger Schneider et édité par André Caquot (1965), puis traduit par Robert Beylot (1991). Mais il fait notamment état d'une correspondance entre Jules Leroy et Pierre Nautin ainsi qu'entre Pierre Nautin et Roger Schneider au sujet de cette découverte, qui montre l'importance des échanges scientifiques dans ce domaine.
} 
dossiers thématiques, mais elle a été numérisée au même titre que les autres archives.

On trouve aussi des carnets de terrain parmi lesquels ceux consacrés à ses études du Nara lors de séjours à Barentu et à Asmara en mai-juin 1967, juin 1968 et 1971. Il s'agit d'enquêtes linguistiques, qui devaient probablement conduire Roger Schneider à publier une description du Nara, mais ce travail n'a jamais vu le jour ${ }^{8}$. Il faut ajouter que des bandes magnétiques audio figurent également dans ces archives et sont des enregistrements de ces mêmes enquêtes linguistiques. Elles ont été confiées à un laboratoire pour être numérisées et ainsi pouvoir être écoutées à nouveau. En tout ce sont cinq bandes qui ont été transformées en sept fichiers .WAV, qui donnent à entendre des locuteurs enregistrés en 1959, 1967 et 1968. Il s'agit là d'une documentation précieuse dans la mesure où l'étude du Nara exige d'enquêter en Érythrée, dans une région aujourd'hui inacessible aux chercheurs. Or les travaux récents de Claude Rilly ont montré le rôle que l'étude du Nara occupe notamment dans la compréhension du méroïtique, langue déchiffrée mais dont le sens échappe encore en grande partie aux savants. D'après ces travaux, le méroïtique appartient à la famille des langues nilo-sahariennes, dont une protolangue, le Soudanique oriental nord peut-être reconstitué à partir d'un vocabulaire commun aux langues de cette famille, que sont le Taman, le Nyimang/Afiti et le Nara9 .

Enfin, le dernier type d'archives concerne le fonds photographique. Celui-ci était globalement conservé dans un carton unique, où figuraient à la fois des négatifs et des développements sur papier. S'agissant des négatifs, on peut d'ores et déjà faire un premier constat: comparé au nombre de sites visités par Roger Schneider, et en particulier le travail réalisé à Gunda Gundēe ${ }^{-10}$ les films négatifs sont très peu nombreux. Ont été numérisés en tout et pour tout sept items différents : le manuscrit du Dersāna Abbā Salāmā ${ }^{11}$, l'évangéliaire de Dabra Libānos de Hām ${ }^{12}$, un rouleau intitulé Gunda Gundē 2 (qui présente quatre peintures réalisées sur parchemin. Ces peintures représentent: Saint Georges, les archanges Kirubēl et Surāfēl, Pierre et Paul, la Vierge à l'enfant ${ }^{13}$ ), un rouleau intitulé Kebra Nagaśt (texte

8 Abraham Johannes Drewes (2002 : 28) en explique ainsi les raisons : "Aux alentours de 1965, il se rend à deux reprises en Éryhrée, à Barentu, pour enquêter sur la langue Néra (Baréa). Mais il ne peut achever ce projet. Au début de sa seconde visite, il est prié de rejoindre Addis Ababa. Cependant à cette exception près, il peut poursuivre ses enquêtes avec fruit ».

9 Rilly, 2010.

10 Schneider, 1963.

11 Il s'agit du Gadla Abbā Salämā, dont la copie lui a été fournie par Georg Gerster. Roger Schneider a publié un résumé de ce texte (1987), sur lequel il portait un regard très critique : "C'est du feuilleton hagiographique de la pire espèce: tous les poncifs, toutes les expressions clichées répétées interminablement, un verbiage qui tourne à vide et n'en finit pas, une imagination décousue, des miracles stupéfiants, voire saugrenus et qu'on retrouve souvent dans des gädlat de la même espèce. Des légendes peuvent être non seulement agréables à lire, mais aussi être instructives ; tout dépend de l'auteur. Ici il est nul, et sa lecture fastidieuse » (1987: 155).

12 Pour plus de détails sur cet évangéliaire et les photographies réalisées par Roger Schneider, voir Derat, 2010.

13 Cette peinture de la Vierge à l'Enfant a été publiée, à partir de clichés personnels, par Gigar Tesfaye (1979). Il publie également une autre peinture, provenant du même manuscrit, les petits Prophètes, 


\section{Marie-Laure Derat}

incomplet), un psautier de Dabra Berhān (mais la région n'est pas précisée), des inscriptions de Abdi Kawehi et d'autres inscriptions dont la provenance demanderait un travail d'identification qui n'a pas encore été fait. Une dernière précision: ces films, anciens, offrent une qualité médiocre parce qu'ils sont décolorés.

La rareté des films négatifs s'explique aisément par la manière dont Roger Schneider concevait son travail. Il a beaucoup partagé avec d'autres chercheurs les données qu'il avait pu recueillir au cours de ses terrains. Ce qui explique l'absence de tous les négatifs des manuscrits de Gunda Gundē, notamment, puisqu'il avait donné ce fonds, ou une partie de celui-ci, à Stefan Strelcyn qui l'a déposé au département d'études sémitiques de l'Institut d'études orientales de l'Université de Varsovie. Stefan Strelcyn a ensuite supervisé des travaux d'étudiants portant sur l'analyse des manuscrits de Gunda Gundēe ${ }^{14}$. D'autres textes trouvés à Gunda Gundē ont été étudiés par André Caquot ${ }^{15}$ ou Robert Beylot ${ }^{16}$.

À partir de ses films négatifs, Roger Schneider avait fait de nombreux tirages papier. Ceux-ci ont été également numérisés. Si bien que pour reprendre le cas de Gunda Gundē, dans le dossier d'archives correspondant, on trouve : le Gadla Abuna Bartalomèwos $^{17}$, le Gadla Banādlewos (GG 62) ${ }^{18}$, le Gadla Esțifanos ${ }^{19}$, le Gadla Mazgaba Śellāsè (GG3) ${ }^{20}$, les Actes des Pères et Frères de Dabra Garzen (GG14) ${ }^{21}$, une Homélie d'Éphrem le Syrien sur Abraham et Sara (GG67)22, un Commentaire de la Genèse (GG

qui ne se trouve pas dans les photographies prises par Roger Schneider. Gigar Tesfaye précise qu'il s'agit d'un manuscrit du Gebra Hemāmāt (Lectionnaire pour la semaine sainte) de l'église d'Aśra Mēṭrā, située à deux jours et demi de marche de Gunda Gundē, dépendante du monastère stéphanite.

14 Termińska, $2006: 271$, note 13.

15 Voir notes 7 et 15.

16 Beylot, 1981.

17 Photographié à Gunda Gundē, ce manuscrit ne fait pas partie de la collection vue par Roger Schneider. Il s'agit en fait d'une copie réalisée par un étudiant de l'Université Paris-I, Thomas Drouin, en 1997, qui l'a adressée à Roger Schneider. Le Gadla Abuna Bartalomèwos occupe 32 folios de l'un des manuscrits du Gadla Ahaw de Gunda Gundē (Drouin, 1998 : 5). Les 32 folios en question figurent au complet dans les archives Roger Schneider.

18 On ne trouve que des notes de la main de Roger Schneider dans ce dossier, avec quelques passages traduits du ge'ez et l'information selon laquelle ces actes ont été édités et traduits par Osvaldo Raineri (1998).

19 Il s'agit de notes prises à la lecture du manuscrit. Les feuillets sont très endommagés et une partie des notes est illisible. Le Gadla Esțifanos a été édité et traduit récemment par Getatchew Haile (2006).

20 Il est difficile de dire, sans faire une étude complète de toutes les photographies du dossier, si la reproduction du manuscrit est complète, mais de nombreux folios figurent dans les tirages papier, ainsi que quelques notes de la main de Roger Schneider.

21 Il ne s'agit pas du texte édité et traduit plus tard par Robert Beylot (1990) à partir du manuscrit EMML 4. Dans le dossier de Roger Schneider, la copie du manuscrit n'est pas complète : ne figurent que 12 folios.

22 Manuscrit étudié par André Caquot, qui a fourni une édition et une traduction de cette homélie à partir de la copie conservée à Gunda Gundē mais aussi à partir d'un autre manuscrit trouvé par Roger Schneider, dans l'église de Dabra Șeyon (1988 : 173). Dans le dossier de Roger Schneider, on trouve la copie de 16 folios du texte. 
33) ${ }^{23}$, le Gadla Tawalda Madhen (GG37) ${ }^{24}$, le Gadla Yesḥaq (GG41)25, les Miracles $d^{\prime} E$ stififanos (GG135) ${ }^{26}$, une Homélie sur la Vierge (GG 185)27, une liste des livres de

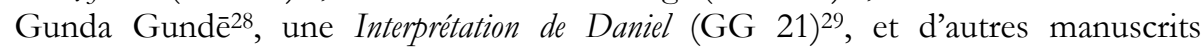
uniquement répertoriés par leur numéro d'inventaire: GG28 (Haymānota Abaw), GG193 (Malke'a Śelläse) et des fragments de manuscrits proprement dits (et non pas leur photo), folios isolés, rapportés par Roger Schneider du monastère de Gunda Gundē.

\section{Conclusion}

Roger Schneider a peu publié en regard des travaux qu'il avait mis en route sur sa table de travail. Parmi ces travaux, on peut notamment citer un article très avancé sur le monastère de Gunda Gundē, qui n'a semble-t-il jamais été publié. Intitulé «Le couvent de Käswa-Gundä Gunde. Fondation, milieu, environnement », cet article étudie le moment de la fondation du monastère à partir des actes de Yeshaq, des miracles d'Esțifanos et d'un recueil de textes ayant trait à l'histoire du monastère, copié par un jeune moine de Gunda Gundē pour Roger Schneider ${ }^{30}$. Dans cet article inédit, Roger Schneider annonce l'édition du Gadla Yesḥaq à partir du manuscrit qu'il avait photographié à Gunda Gundē et qu'il avait répertorié sous le numéro 36. L'un des dossiers composant les archives de Roger Schneider porte le titre de Gadla Yeshaq (Agame). Il contient une copie, à la main, du texte du manuscrit Gunda Gundē 36 (du fol. 55r au fol. 90r) ${ }^{31}$.

Concernant le Gadla Yeshaq, on trouve également dans les archives un dossier contenant des notes prises sur le manuscrit GG 41, lui aussi intitulé par Roger Schneider Gadla Yesḥaq (Agame). Il semble qu'il avait préparé une brève présentation de ce texte, qu'il devait sans doute adresser à l'un de ses correspondants mais bien

${ }^{23}$ En plus des clichés d'une partie du manuscrit (fol. 1-11r; 146r-150r), on trouve une traduction des huit premiers folios réalisée par Roger Schneider. Il faut préciser ici qu'il ne s'agit pas du Livre des Mystères du Ciel et de la Terre, qui glose également sur la Genèse, et dont on sait que Roger Schneider en avait trouvé une copie à Gunda Gundē (Anfray, $2003: 368$ ).

${ }^{24}$ Le dossier ne contient que les huit premiers folios en tirage papier.

${ }^{25}$ Dans ce cas, il s'agit de notes prises par Roger Schneider sur le manuscrit lui-même mais pas des photographies du manuscrit.

${ }^{26}$ Le manuscrit parait très endommagé sur les photos, seuls six folios sont présents. Roger Schneider a recopié à la main les fols. $18 \mathrm{r}$ à $31 \mathrm{v}$, correspondant à un texte intitulé «Miracle de Käswa » par les moines de Gunda Gundē.

${ }^{27}$ Le texte, attribué à Minās, évêque d'Aksum, parât complet, il compte 12 folios et est intégré dans un manuscrit rassemblant des homélies dédiées à Marie, puisque le texte qui suit est celui qui doit être lu pour la fête de la naissance de la Vierge.

28 Cette liste compte cinq folios et dénombre 143 manuscrits.

${ }^{29}$ Seuls deux folios de ce manuscrit figurent dans les archives.

${ }^{30}$ Cet article figure aux pages 115-123 de ce volume.

${ }^{31}$ Nous pouvons ici faire état d'un problème dans la numérotation des manuscrits de Gunda Gundē. Dans les notes de Roger Schneider, le Gadla Yeshaq (Agamé) est répertorié au numéro 36. Mais on trouve aussi un dossier intitulé Yeshaq Agamé qui renvoie à un manuscrit numéroté GG 41. Vraisemblablement il s'agit de deux mêmes textes, mais dans la mesure où le manuscrit GG 41 fait seulement l'objet de notes, sans donner à voir le texte, il est difficile de résoudre cette question. 


\section{Marie-Laure Derat}

évidemment, dans ses archives, ne figurent pas la lettre elle-même et donc le destinataire, seulement son brouillon. On y retrouve notre savant et sa rigueur : «Le copiste était lamentable, au point qu'on se demande parfois s'il connaissait le guèze. Les fautes fourmillent et les points de séparation sont mis de façon absolument fantaisistes. J'ai parcouru le texte rapidement et voici les renseignements que je peux vous fournir $[\ldots] »$.

Ces archives sont donc une mine d'informations inappréciables et on ne peut que se féliciter de leur mise à disposition à l'Institute of Ethiopian Studies. Au-delà de Roger Schneider, la question des archives de chercheurs doit être une préoccupation. Les nombreux projets d'inventaires en cours aujourd'hui pourraient intégrer avec profit un volet sur les archives des chercheurs qui ont travaillé de nombreuses années en Éthiopie et en Érythrée. Ces archives complèteraient sans aucun doute la documentation actuelle, de nombreuses pièces, manuscrits, peintures, inscriptions étant soit inaccessibles soit disparues.

Dans cette perspective, le Centre français des études éthiopiennes tente de retrouver et de mettre en valeur les archives de la mission archéologique française, son ancêtre. Cette mission s'est éteinte en laissant derrière elle une bibliothèque et des fonds d'archive d'un grand intérêt puisqu'ils documentent une trentaine d'années de recherches. Récemment, la bibliothèque de la section d'archéologie a été réintégrée au fonds de la bibliothèque de l'ARCCH et est désormais consultable au musée national d'Addis Abeba. Une partie de la photothèque de cette section est également conservée au musée national. Nous espérons que d'autres fonds seront prochainement mis en valeur et accessibles à tous.

\section{Inventaire des archives numérisées de Roger Schneider ${ }^{32}$}

Pictures of manuscripts in Ethiopian churches and monasteries:

- Dersāna Abbā Salāmā

- $\quad$ Gospel of Dabra Libānos (Hām)

- Gunda Gundē 2

- Kebra Nagaśt

- Mazmur Dabra Berhān

Southarabic and ge'ez inscriptions

- $\quad$ Abdi Kawehi

- Various inscriptions

Archives scanned

abba Tewelde Medhin: articles of abba Tewelde Medhin on rupestrian churches in Tigray

- Abuna Pantalewon inscription

- African arts festival: article of Roger Schneider "Overview on the Ethiopian Antiquity"

- Aksum: 2 files (Aksum I and Aksum II) - notes on secondary studies concerning Aksum and aksumite inscriptions; drafts of Roger Schneider on aksumite inscriptions.

- Annals (Addi Neamin, Bizan): notes on the Annals, copy of the ge'ez text, translation into french.

32 Cet inventaire est présenté en anglais car c'est dans cette langue qu'il a été effectué afin de rendre ces archives accessibles à des non francophones. 


\section{Les archives Roger Schneider}

- $\quad$ Arkalades: notes on the Conti Rossini' study on Arkalades

- Armachaho monasteries: notes on Abbadie 165 on the monasteries of Armachaho

- $\quad$ Badi': notes on Badi', Bedja name of Massawa.

- $\quad$ Bafaqih (M.A.): notes on an interpretation of Bafaqih on Du. I/ Y.G.R.W.

- Baql: notes on the term "baql", mule/horse

- Barentu: 4 fieldwork books with vocabulary on Nara language (Barentu, may 1967; Asmara, may 1967; Asmara, may-june 1967; Barentu, june 1968; Asmara 1971).

- $\quad$ Barya: extract from the unpublished notes of d'Abbadie on Barya; notes of Cerulli on Barya

- Besu'a Amlak: notes on Besu'a Amlak

- $\quad$ Beta Lehem church: amharic article on the church of Betalehem (Gaynt)

- $\quad$ BNF Ethiopien d'Abbadie 116: print film of the manuscript BNF Ethiopien d'Abbadie 116.

- $\quad$ Brass roundel Aksum: study by Schneider of the brass-roundel discovered by Phillipson in Aksum.

- $\quad$ Bruce Judith: notes from James Bruce, on Judith

- Churches on lake Tana: fieldwork book of a trip of Roger Schneider in the churches of Lake Tana.

- $\quad$ Cross: picture of a manuscript (unidentified)

- Dabra Libanos-Ham: notes of Roger Schneider on the documents of the Gospel of Ham (copy of the ge'ez text, translation into english...), pictures of the Gospel of Ham

- Daga-Fere Seyon: note of Fere Seyon on the painting of Daga

- Dahlak: pictures of the Dahlak islands

- Danba Mess: documents from the Gospel of Danba Mess (copies of the ge'ez text)

- Dersana Ephrem: notes from a manuscript of Dabra Seyon, Geralta

- Djibil Habasi: notes on this family name

- Documents from Dabra Endreyas: notes from Conti Rossini and corrections added by Schneider.

- Documents from various churches: acts copied on different manuscripts with translation and notes of Schneider.

- Enda Qirqos: pictures of the site

- Exhibition BN Paris: copy of the catalogue of the exhibition organised by Eric Godet at the National Library in Paris with a letter from Eric Godet to Roger Schneider.

- $\quad$ Expedition to Tana (Wright): Report of S. Wright on his expedition to Lake Tana.

- $\quad$ Field trips: documents regarding field trips of Roger Schneider (funds...).

- Gabra Krestos of Dabra Maryam: notes from a "Gospel of Sayfa Ar'ad”.

- Gadla Akala Krestos: pictures of a manuscript with the Gadla Akala Krestos from Medr Zoga.

- Gadla Bartalomewos of Jammadu: pictures of the manuscript, notes and translation of Roger Schneider.

- $\quad$ Gadla Yeshaq (Agame): copy of the ge'ez text (Gunda Gundē 36).

- Gadla Yostinos of Dabra Maryam: translation and notes from a manuscript of Dabra Maryam of Qohayn.

- $\quad$ Gadla Zamadhen (Zazya): pictures of a manuscript (unidentified origin)

- $\quad$ Gerster pictures: print films of pictures made by Georg Gerster

- Gondar: pictures of sites in the surroundings of Gondar

- Gospel commentaries: commentaries from the manuscripts Eth. 65, Abb. 191, Abb. 24, Abb. 130.

- $\quad$ Gospel of Dabra Maryam: some pictures of the Gospel of Dabra Maryam.

- GRBYN: notes on the term GRBYN

- Gunda Gunde manuscripts: Gadla Abuna Bartalomewos, Gadla Banadlewos, Gadla Estifanos, GG3 Mazgaba Sellasse, GG14 Fathers of Dabra Garzen, GG 28, GG 33 Genesis, GG 37 Gadla Tawalda Madhen, GG 41 Yeshaq, GG 67, GG 135 Miracles of Estifanos, GG 185 Homily on the Virgin, GG 193, GG list ob books, Käswa Gunda Gunde, GG 21 Tergwam Daniel, GG fragments.

- $\quad$ Hahn (W.): correspondance with Hahn

- $\quad$ Hippolyte on Antichrist: notes from different secondary studies

- Inscriptions: DAE 8; Ezana inscription; J.E. 01, J.E. 02; DAE 4, 6, 7; inscription 111-112; RIE 239; diverse inscriptions

- Kebran: Gadla Zayohannes of Kebran (pictures of the manuscript); Gospel of Kebran (some pictures of the manuscript); history of the building of the church of Kebran (pictures of the manuscript).

- $\quad$ Language survey of Ethiopia: questionnaire sent to Schneider by Bender

- Manuscripts of Dabra Bizan: pictures of the Mashafa Hasusan, documents, annals... 


\section{Marie-Laure Derat}

- Manuscript fragments: fragments from Guh, fragments classified by Schneider according to the datation of the handwriting.

- $\quad$ Manuscript miscellanous: pictures of various manuscripts without indentification.

- Manuscripts of the Archaeological Mission: copy of the text of the manuscripts' fragments belonging to the French Archaeological mission.

- Maps of Lalibela: from the publication of Monti della Corte

- Mashafa Mestir ZaHepolitos: pictures of a manuscript (unidentified origin)

- Matara: tables for a publication of the archaeological mission on Matara; pictures of artefacts from Matara.

- Miscellaneous notes: notes in the hand of Roger Schneider found in disorder (out of their file)

- $\quad$ MKRB: notes on the meaning of the term MKRB

- $\quad$ Muller (W.W.): correspondance with Muller

- Numerals: synthesis on the numerals in Greek language and Ethiopic language.

- Old Ethiopian inscriptions (tablets): synthesis made by a student named Derege Girma.

- Phillipson (D.W.): correspondance with Phillipson

- $\quad$ Pictures of Gerster: copies of pictures taken by Georg Gerster.

- Pictures of sites and churches (miscellania): the sites and churches are not identified in the back of the pictures themselves.

- Prechristian aksumite epigraphy: notes on symbols and divinities in the prechristian aksumite epigraphy.

- $\quad$ Print films of edited studies: A.G. Lundin; Geography of A. d'Abbadie; Conti Rossini - Abba Afse; Esteves Pereira - Abba Yeshaq of Tiphre; Historia Iemanae; Conti Rossini - Ham; BNF Exhibition catalogue; Conti Rossini - Appunti sulla lingua khamta dell'Averghellè; Conti Rossini Studi su popolazioni dell'Etiopia, Sahel Eritreo; Conti Rossini - Saggio sulla toponomastica dell'Eritrea tigrigna; Races and tribes of Eritrea; Tubiana - Les noms de Gondar et d'Aksum; Wiet - Roitelets de Dahlak.

- Psalm book: notes on psalms and psalter.

- Psalm seqwar introduction: introduction to the psalter, pictures of a manuscript (origin unidentified).

- $\quad$ Radiocarbon datation: results of radiocarbon datation on samples from Yeha and Matara.

- Rupestrian churches: report of a first mission to Tigre on the rupestrian churches.

- Sabean inscriptions: article of Roger Schneider submitted for the "Mélanges David Cohen" on "Remarques sur les inscriptions sabéennes de l'Éthiopie préaksumite".

- Salam and Malke'a: notes on different terms in ge'ez

- $\quad$ Schneider drafts: notes on vocabulary, drafts of articles...

- Ser'ata Mahlet: pictures of a manuscript (origin unidentified)

- $\quad$ Simeone-Senelle (M.C.): correspondance with M.C. Simeone-Senelle.

- $\quad$ Southarabic documents (Marcel Cohen): autocopy

- Synaxary: notes from various secondary sources

- Takasta Berhan Dima: notes on Takasta Berhan (monastic genealogy)

- Tentative etymology of ancient names \& Kunama (Nicodemus Idris): secondary study written by Nicodemus Idris (hand-writting).

- Tergum: synthesis on the teaching of tergum in the Ethiopian church and notes.

- Traditional education: notes gathered under the title "traditional education"

- Vocabulary: index card on vocabulary (nara?)

- Writing: notes, drafts on Ethiopian writing

- $\quad$ Yage'ez qwanqwa sewasew: extract from the book of abba Takla Maryam, Keren, 1899.

- $\quad$ Yaqeddus Yared Zema: pictures of a manuscript from Dabra Berhan

- Yeha: pictures of inscriptions from Yeha

- ZaYohannes of Kebran: Madeleine Schneider draft and correspondance with the Corpus Scriptorum Christianorum Orientalium.

\section{Bibliographie}

Anfray F., 2002, Roger Schneider, Rassegna di Studi Etiopici, 1 (1), 147-150. 


\section{Les archives Roger Schneider}

Anfray F., 2003, Roger Schneider. Luxembourg, 29 avril 1917-Addis Abeba, 4 avril 2002, Annales d'Ethiopie, 19, 367-369.

Bernand E., Drewes J.A. \& Schneider R., 1991, Recueil des inscriptions de l'Éthiopie des périodes pré-axoumite et axoumite, t. 1 : les documents ; t. 2 : les planches, Paris, De Boccard.

Bernand E., 2000, Recueil des inscriptions de l'Éthiopie des périodes pré-axoumite et axoumite, t. 3: traductions et commentaires. A, les inscriptions grecques, Paris, De Boccard.

Beylot R., 1981, Estifanos, hétérodoxe éthiopien du XVe siècle, Revue de l'histoire des religions, 198 (3), 279-284.

Beylot R., 1990, Actes des Pères et Frères de Debra Garzen : introduction et instructions spirituelles et théologiques d'Estifanos, Annales d'Éthiopie, 15, 7-49.

Beylot R., 1991, Hippolyte de Rome, "Traité de l'Antéchrist » traduit de l'éthiopien, Semitica, 40, 107-139.

Caquot A., 1965, Une version ge'ez du traité d'Hippolyte de Rome sur l'Antichrist, Annales d'Éthiopie, 6, 165-214.

Caquot A., 1988, Une homélie éthiopienne attribuée à Saint Mar Éphrem sur le séjour d'Abraham et Sara en Égypte, in Coquin R.G. (éd.), Mélanges Antoine Guillaumont: contributions à l'étude des christianismes orientaux, Genève, Patrick Cramer (Cahiers d'orientalisme, 20), 137-185.

Drewes A.J., 2002, In memoriam Roger Schneider (1917-2002), Aethiopica, 5, 206214.

Drouin T., 1998, Les listes et le gadla Abuna Bartalomewos : deux types de documents pour une histoire des métropolites d'Éthiopie, Mémoire de DEA, Université Paris-I.

Getatchew Haile, 2006, The ge'ez acts of abba Estifanos of $G^{w}$ endag'ende, Louvain (CSCO 619-620, Script. Aeth. 110-111).

Gigar Tesfaye, 1979, Notes sur Gunda-Gundié, Abbay, 10, 93-100.

Kebbede Mikael \& Leclant J., 1955, La section d'archéologie (1952-1955), Annales d'Éthiopie, 1, 1-8.

Leclant J., 2002, Rapport sur l'état des publications de l'Académie pour l'année 2001, Comptes-rendues des séances de l'Académie des Inscriptions et Belles-Lettres, 146 (1), 287-294.

Raineri O., 1998, Atti di Banadlewos (1303-1400), Turnhout, Brepols (Patrologia Orientalis, 48.1).

Rilly C., 2010, Le méroïtique et sa famille linguistique, Paris-Louvain, Peeters (coll. SELAF, 454, Afrique et langage, 14).

Schneider R., 1963, La bibliothèque du couvent de Gunda Gundé, Tarik, 2, 45-46.

Schneider R., 1987, Les actes d'Abuna Salama, Annales d'Éthiopie, 14, 153-172.

Termińska K., 2006, The trinatarian incipit in the the Context of Ethiopia's Heretical Discursive Formation in the period from the $15^{\text {th }}$ to the $17^{\text {th }}$ century, in Witakowski W. \& Lykowska L. (ed.), Wälättä Yohanna. Ethiopian Studies in 


\section{Marie-Laure Derat}

Honour of Joanna Mantel-Niecke on the occasion of the 50th year of her work at the Institute of Oriental Studies, Warsaw University, Varsovie (Rocznik Orientalistyczny, 59), 265-280.

\section{Résumé/Abstract}

Derat M.-L., 2011, Les archives Roger Schneider (1917-2002) au centre Walda Masqal (Institute of Ethiopian Studies, Addis Abeba), Annales d'Éthiopie, 26, 291-302.

Les archives Roger Schneider sont aujourd'hui consultables au centre Walda Masqal, centre d'archives de I'Institute of Ethiopian Studies à Addis Abeba. Cet article présente le fonds documentaire, aussi bien la bibliothèque donnée par la famille de Roger Schneider, que les archives du savant, qui ont été numérisées dans le cadre d'un projet conjoint au Centre Français des Études Éthiopiennes et à l'Institute of Ethiopian Studies, afin de les sauvegarder.

Mots-clefs : Roger Schneider, archives, Gunda Gundē.

The archives of Roger Schneider (1917-2002) in the Walda Masqal Centre (Institute of Ethiopian Studies, Addis Ababa) - The archives of Roger Schneider are now available in the Walda Masqal centre, an archives' centre of the Institute of Ethiopian Studies in Addis Abeba. This article presents the documents of this collection, i.e. the library itself given by the family of Roger Schneider, as well as the archives of this great scholar, which have been digitalised in order to preserve them. This digitalisation was the result of a joint project between the Centre Français des Études Éthiopiennes and the Institute of Ethiopian Studies.

Keywords: Roger Schneider, archives, Gunda Gundē. 